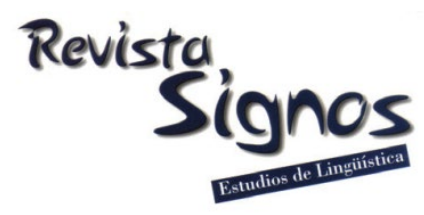

\title{
Agentividad y modo: Grados de responsabilidad en mensajes automáticos
}

\section{Agentivity and Mood: Degrees of responsibility in automatically generated messages}

\author{
María Belén Grisolía \\ UNIVERSIDAD NACIONAL DE MAR DEL PLATA \\ ARGENTINA \\ mbgrisolia@gmail.com
}

Recibido: 25-IX-2019 / Aceptado: 28-VIII-2020

DOI: $10.4067 /$ S0718-09342021000100148

\section{Resumen}

En este trabajo proponemos estudiar textos cada vez más significativos en nuestra cultura: los mensajes automáticos -vía correos electrónicos, mensajes de texto y contestadores telefónicos. El interés por el análisis lingüístico de estas manifestaciones discursivas surge de una serie de testimonios recolectados por la autora que revelan un humor social negativo en relación con ellas; se trata de interacciones socialmente polémicas lo que motiva y justifica el interés por su análisis. La hipótesis sostiene que la sensación, ampliamente extendida entre los hablantes de la comunidad estudiada, de estar participando de 'inter' acciones en las que, finalmente, parecen estar hablando solos se relaciona con la forma en que se distribuyen los grados de responsabilidad en el discurso, responsabilidad que definimos en términos de agentividad y modo. Para trabajar con la hipótesis empleamos la Lingüística Sistémico-Funcional (Halliday, 1994, 2008; Halliday \& Matthiessen, 2004) como teoría gramatical de base, suplementada por un enfoque discursivo estratégico (Menéndez, 2000, 2005). Los resultados confirman la hipótesis: el análisis del corpus muestra altos grados de agentividad asignada al destinatario de los mensajes pero grados muy bajos de agentividad del lado del emisor institucional.

Palabras Clave: Texto, discurso, estrategia, agentividad, modo. 


\begin{abstract}
This paper examines a type of discourse that is becoming increasingly meaningful in our culture: the automated email, text and phone messages. It is part of an ongoing research project regarding the grammatical and discursive constraints of the phenomenon of agentivity. We specifically analyze the effects of agentivity related to negotiation of responsibility in automatically generated messages. We use the Systemic Functional Grammar (Halliday, 1994, 2008; Halliday \& Matthiessen, 2004), supplemented by a discursive strategic approach (Menéndez, 2000, 2005), to analyze how agency is structured and distributed among participants: the institutional sender and the noninstitutional addressee. The results confirm our hypothesis: the strategy is oriented to personalize the Addresse, to whom the responsibility over the predicated actions is pointed. The participant 'sender', on the other hand, is constructed by a discourse strategy oriented to impersonalization.
\end{abstract}

Key Words: Text, discourse, strategy, agentivity, Mood.

\title{
INTRODUCCIÓN
}

'No respondas a este mensaje porque es automático': la comunicación con instituciones y empresas nos enfrenta, en los últimos años, a un nuevo tipo de interacción en el que una de las características definitorias es la restricción, justamente, de la posibilidad de interactuar. El participante no institucional se encuentra, por un lado, con la instrucción de no responder $y$, por otro, con la única alternativa de satisfacer sus inquietudes en el marco de las opciones -muy limitadas- habilitadas por el participante institucional. Nos referiremos a dichos participantes con la categoría 'destinatarios' porque son quienes se encuentran con estos mensajes (cuando los reciben en sus dispositivos electrónicos o los escuchan ante una llamada telefónica) y cuya acción en respuesta es la que se ve restringida en estas interacciones, como en el ejemplo que sigue:

Gracias por comunicarte con el centro de atención al vecino de la municipalidad \si tu llamada es por una emergencia / llamá al 911 \ | si querés saber el número de celular de la patrulla de tu barrio | marcá $9 \backslash$ para solicitar turnos / marcá $1 \backslash$ para hacer un reclamo / marcá $2 \backslash$ para realizar una denuncia marcá $3 \backslash$ para actividades de cultura marcá $4 \backslash$ para saber si tu auto fue remolcado por tránsito / marcá 5 \ para conocer el estado de una solicitud en proceso marcá $6 \backslash$ para escuchar nuevamente las opciones marcá $0 \backslash$

Los asuntos representados en el mensaje anterior son, como vemos, diversos, heterogéneos e involucran distintos grados de urgencia: desde obtener información sobre una actividad de cultura hasta la necesidad de saber si un auto fue remolcado, hacer una denuncia o alertar sobre una emergencia. Las reacciones de los participantes-individuos frente a este tipo de mensajes son inmediatas y contundentes. Nos sentimos limitados, no escuchados, tal como lo atestiguan los numerosos testimonios que la autora ha recopilado a lo largo de los últimos años, entre los cuales 
presentamos apenas uno a continuación. Se trata de un tweet posteado por una clienta de un banco. Su mensaje -consecuencia de la experiencia de interacción frustrada- es lo suficientemente claro: "Dale@BancoGalicia. No pido mucho. Solamente quiero hablar con un ser humano cuando te llamo. Ninguna de las opciones de tu maquinita me responde lo que quiero saber". Las reacciones de sus seguidores también son categóricas. A los 56 minutos de ser publicado el tweet, ya contaba con 66 'me gusta', había sido retuiteado 12 veces y contaba con 12 comentarios de apoyo. No había transcurrido una hora y casi cien personas se habían sentido identificadas con la situación reseñada. En este artículo abordamos el estudio de ese tipo de mensajes automáticos -vía correos electrónicos, mensajes de texto y contestadores telefónicos. El interés por el análisis lingüístico de estas manifestaciones discursivas surge de testimonios como el anterior que revelan, a nuestro juicio, un humor social negativo, de profundo fastidio, en relación con ellas; se trata de interacciones socialmente polémicas lo que, creemos, motiva y justifica el interés por su análisis. Hasta el momento, no hemos encontrado estudios que se dediquen específicamente a analizar estos textos y a interpretar sus efectos discursivos. En este sentido confiamos en que esta investigación puede ser una primera contribución a explorar desde una perspectiva gramatical y discursiva mensajes cada vez más relevantes en las sociedades actuales. La hipótesis de partida sostiene que la sensación, ampliamente extendida entre los hablantes de la comunidad estudiada, de estar participando de 'inter'acciones en las que, finalmente, quedan hablando solos se relaciona con la forma en que se distribuyen los grados de responsabilidad en los mensajes automáticos, responsabilidad que definiremos en las próximas secciones en términos de agentividad y modo. De acuerdo con la hipótesis, dicho efecto se explica por altos grados de agentividad asignada al destinatario de los mensajes pero grados muy bajos de agentividad del lado del emisor institucional. Para probar la hipótesis, analizamos cómo se representan las acciones predicadas en dichos mensajes automáticos y quiénes son los señalados como encargados de llevarlas a cabo. En las próximas secciones presentaremos, primero, el marco teórico gramatical y discursivo-estratégico en el que se encuadra el análisis textual propuesto y su posterior interpretación discursiva, seguido de la descripción del marco metodológico empleado y el análisis de tres casos testigo del corpus.

\section{Marco teórico}

Para trabajar con la hipótesis empleamos la Lingüística Sistémico-Funcional (Halliday, 1994, 2008; Halliday \& Matthiessen, 1997, 2004 -de aquí en más LSF) como teoría gramatical de base, suplementada por un enfoque discursivo-estratégico (Menéndez, 2000, 2005). Comenzamos por establecer una distinción teórico-analítica entre texto y discurso. Lavandera (2014 [1992]: 348) propone la siguiente ecuación: Discurso $=$ Texto + Contexto. Según ella: 
“el texto está hecho de elementos y relaciones expresadas lingüísticamente. El discurso incorpora los elementos y las relaciones extralingüísticas que se manejan en la producción del texto".

En esta misma dirección, Menéndez (2005) vincula la descripción de los recursos a la dimensión textual y el análisis de su combinación en estrategias a la interpretación discursiva:

"El texto es una unidad gramatical conformada por un conjunto de recursos, es decir, de opciones efectivamente seleccionadas. El discurso es una unidad estratégico-pragmática. En consecuencia, los recursos gramaticales son los elementos puntuales que conforman una estrategia discursiva. Permiten que esta se constituya como tal. Los recursos, por lo tanto, permiten la descripción gramatical. Su combinación estratégica, la explicación e interpretación discursivas" (Méndez, 2005: 2).

En dicho marco encuadramos nuestro trabajo: analizamos los recursos en el texto usando la LSF como teoría gramatical de base, primero, para interpretar, después, su combinación en estrategias discursivas. La LSF concibe la gramática en términos funcionales, como un sistema de opciones disponibles que los hablantes actualizamos toda vez que producimos textos. En dicha línea, prestamos especial atención al sistema y el texto simultáneamente: pensar en sistemas de opciones disponibles permite observar sobre la base de qué opciones potenciales (que conforman el sistema) se recortan las opciones efectivamente realizadas (que conforman los textos); esto hace posible establecer qué pudo haber sido y no fue, y determinar por qué fue de una manera y no de otra. Dicho de otro modo, permite dar respuesta a la pregunta funcionalista por excelencia: por qué el hablante dijo lo que dijo de una manera y no de otra, lo cual ofrece argumentos para la interpretación discursiva del alcance de las opciones intercambiadas y de sus repercusiones sociales. Nuestro objetivo es explicar discursivamente ciertas elecciones que realizan los hablantes/escritores en función de los grados de responsabilidad que construyen y negocian en la interacción. Para eso, establecemos un vínculo entre las realizaciones de los sistemas de transitividad y modo, sistemas que codifican los significados experiencial e interpersonal respectivamente (Halliday, 1994, 2008; Eggins, 2004; Halliday \& Matthiessen, 1997, 2004; Ghio \& Fernández, 2008; Halliday \& Webster, 2009; Lavid, Arús \& Zamorano, 2010) con el objetivo de identificar y definir estrategias discursivas orientadas al establecimiento de grados particulares de responsabilidad entre los participantes de las interacciones que conforman el corpus. En las siguientes subsecciones nos detendremos en la descripción del sistema de transitividad, vinculado con la noción de agentividad, y del sistemade modo. 


\subsection{Responsabilidad - Agentividad}

El elemento central del sistema de transitividad es el proceso, realizado típicamente por los verbos. Dos son los modelos que delinean el sistema de transitividad, el transitivo y el ergativo: "the two models complement one another (...) they embody different generalizations about the flux of experience, resonating with different situation types" (Halliday \& Matthiessen, 2004: 285). Desde el punto de vista del modelo transitivo, el foco está puesto en lo que diferencia a los procesos y los hace formar parte de distintos tipos. El modelo ergativo, por su parte, representa una interpretación generalizadora, que atraviesa los distintos tipos de procesos; dicho de otro modo, se concentra en lo que los procesos tienen en común: la estructura proceso + medio ( \pm agente) (Halliday \& Matthiessen, 2004).

Según el modelo transitivo, los distintos tipos de procesos exigirán roles específicos que representan el papel que personas, objetos, fenómenos, etc. desempeñan en el proceso en cuestión de acuerdo con su significado experiencial (Halliday, 1994; Halliday \& Matthiessen, 2004). De los seis tipos de procesos que configuran el modelo transitivo nos interesan particularmente los procesos materiales, conductuales, verbales y mentales/sensoriales pues es posible identificar en ellos un participante ${ }^{1}$ activo que hace (en el caso de los procesos materiales y conductuales), dice (en los procesos verbales), piensa, reacciona o siente (en el caso de los procesos mentales/sensoriales). Frente a tales procesos, en los relacionales y existenciales encontramos un participante que es caracterizado, identificado o cuya existencia se marca a partir del proceso pero que no resulta activo en ninguno de los sentidos que señalamos antes. Sostenemos, entonces, que la agentividad está en juego especialmente en esos cuatro tipos de procesos: ${ }^{2}$ la actividad de dicho participante puede ser material (en el caso del actor y el conductor), verbal (dicente) o mental, sensorial (procesador) como corresponde a los distintos tipos. Es importante aclarar que la actividad mental/sensorial -a diferencia de la material y la verbal- es intransferible e involuntaria: ${ }^{3}$ el procesador no hace pero sí experimenta (percibe, reacciona, conoce o siente) un fenómeno. En este sentido entendemos que sigue participando de una actividad, aunque en términos de una reacción no voluntaria. El principio de gradualidad (Menéndez, 2013) permite explicar la organización de los procesos a lo largo de un continum definido a partir de rasgos que contemplan el grado de concreción del proceso y su grado de agentividad. La siguiente tabla muestra los distintos tipos de procesos que involucran agentividad, organizados desde el mayor (IV) al menor (I) grado de concreción. 
Tabla 1. Tipos de procesos que realizan actividad y participantes activos (elaboración propia basada en Halliday \& Matthiessen (2004, 2014) y Menéndez (2013)).

\begin{tabular}{|c|c|c|}
\hline Tipo de proceso & Participante activo & $\begin{array}{c}\text { Ejemplos } \\
\text { (tomados del corpus que } \\
\text { presentaremos en la próxima sección) }\end{array}$ \\
\hline $\begin{array}{l}\text { MATERIAL (grado de } \\
\text { concreción: IV) } \\
\text { Ej. Hacer la fila }\end{array}$ & $\begin{array}{l}\text { ACTOR [+ejecutor + causa }] \\
\text { EJECUTOR }[+ \text { ejecutor - } \\
\text { causa }]\end{array}$ & $\begin{array}{l}\text { (1) Descargá Whyline y hacé fila de manera } \\
\text { virtual (B-10) } \\
\text { (2) Whyline hace la fila por vos. (B-10) }\end{array}$ \\
\hline $\begin{array}{l}\text { CONDUCTUAL (grado } \\
\text { de concreción: III) } \\
\text { Ej. Decidir }\end{array}$ & $\begin{array}{l}\text { CONDUCTOR } \\
\text { (ser con consciencia) }\end{array}$ & (3) Decidimos multiplicar las ofertas (A-20) \\
\hline $\begin{array}{l}\text { VERBAL (grado de } \\
\text { concreción: II) } \\
\text { Ej. Informar }\end{array}$ & $\begin{array}{l}\begin{array}{l}\text { DICENTE [+transmisor } \\
+ \text { fuente] }\end{array} \\
\text { TRANSMISOR [+transmisor } \\
\text {-fuente] }\end{array}$ & $\begin{array}{l}\text { (4) Informamos a ustedes que nuestro nuevo } \\
\text { borario de atención será de } 10 \text { a } 14 \text { (B-8) } \\
\text { (5) Desde abora, nuestro Newsletter te } \\
\text { informará los últimos Groupones (A- 20) }\end{array}$ \\
\hline $\begin{array}{l}\text { MENTAL-SENSORIAL } \\
\text { (grado de concreción: I) } \\
\text { Ej. Sentir (con el } \\
\text { significado de lamentar) }\end{array}$ & $\begin{array}{l}\text { PROCESADOR } \\
\text { (ser con consciencia) }\end{array}$ & $\begin{array}{l}\text { (6) Lo sentimos mucho (se retrasó la entrega) (A- } \\
\text { 14) }\end{array}$ \\
\hline
\end{tabular}

Los procesos materiales, del tipo de 'descargar' o 'hacer fila' (que aparecen en los ejemplos (1) y (2) de la Tabla 1), representan el mundo exterior del 'hacer' por lo cual se ubican cerca del polo [+concreto] en la escala del grado de concreción. El participante ACTOR, que hace, se caracteriza por ser una entidad potente animada, humana y voluntaria: en el rol ACTOR, entonces, el participante que causa el proceso y el que lo ejecuta coinciden (como en el caso del ejemplo 1). El rol de EJECUTOR, por su parte, se define como el participante que concreta materialmente la acción en nombre de otro (agente o causa) que la instiga, la provoca. ${ }^{4}$ Los EJECUTORES pueden ser [ \pm humanos], [ \pm animados] pero siempre son [-voluntarios] en relación con la actividad material que ejecutan (como muestra el ejemplo 2 en el que una aplicación [-animado] [-voluntario]'hace la fila'). Los procesos de conducta tienen un grado de concreción menor que el de los materiales: representan la esfera del 'hacer' pero participan también rasgos mentales o sensoriales. Halliday y Matthiessen (2004) definen los procesos de conducta como manifestaciones externas de procesos internos, en este sentido los CONDUCTORES son seres con consciencia, [+humanos] y [+voluntarios], y siempre están en la causa del proceso (como el participante que toma la decisión en el ejemplo 3). Los procesos verbales son definidos como intercambios simbólicos de significados, esto es, relaciones simbólicas construidas en la consciencia humana y ejecutadas en la forma de lenguaje (Halliday \& Matthiessen, 2004). Muchos de ellos constituyen, en rigor, formas de comportamiento. Les asignamos el número II de nuestra escala en tanto dicho comportamiento no es material sino verbal, simbólico. Definimos el DICENTE como 
el participante en el que el transmisor, el que dice, constituye también la fuente de lo que dice (como se ve en el ejemplo 4). Cuando transmisor y fuente no coinciden, el participante que dice se constituye como un mero TRANSMISOR verbal. Los TRANSMISORES pueden ser [ \pm humanos], [ \pm animados] pero son [-voluntarios] (en tanto ejecutan, diciendo, la acción verbal cuya fuente es otro participante, como se observa en el ejemplo 5: el Newsletter [-animado] [-voluntario]'informará' aquello para lo que fue programado). Importa destacar la diferencia entre el participante que ejecuta la actividad (que hace o dice) y el que la causa (que es la fuente del hacer o del decir) en los procesos materiales y verbales ya que esta diferencia es fundamental a la hora de observar grados de responsabilidad discursiva. En el extremo [-concreto] de la escala de actividad ubicamos a los procesos mentales/sensoriales, procesos que representan el mundo interior de la consciencia y, por lo tanto, definen un PROCESADOR. Halliday y Matthiessen (2004: 250) señalan que "behaver is typically a conscious being, like the Senser; the Process is grammatically more like one of 'doing". La diferencia entre el CONDUCTOR y el PROCESADOR radica, entonces, en la naturaleza de la actividad de la que forman parte: el procesador, a diferencia del conductor, no hace nada sino que experimenta, percibe -i.e. le sucede- un fenómeno (como muestra el ejemplo 6). El modelo ergativo, por su parte, representa una interpretación generalizadora (Halliday \& Matthiessen, 2004); el punto de vista ergativo observa si el proceso se produce 'desde dentro' o 'desde fuera', es decir, el foco está puesto en la causa: de acuerdo con la estructura ergativa, en todo proceso aparece un participante involucrado, el medio, sin el cual el proceso no podría suceder; ahora bien, la pregunta es si el proceso es producido por ese participante o por alguna otra entidad externa al proceso mismo, en cuyo caso hablaríamos de un agente: en palabras de Halliday y Matthiessen (2004: 290), "either the process is represented as self-engendering (in which case there is no separate agent) or it is represented as engendered from outside (agent)". La interpretación ergativa constituye una explicación complementaria a la transitiva en la que se observa, entonces, la causa que subyace en un determinado proceso y se distingue el agente del medio que lo hace posible (Halliday, 1994). La consideración complementaria de ambas interpretaciones del sistema de transitividad, i.e. la transitiva y la ergativa, resulta fundamental puestos a observar la construcción y distribución de grados de responsabilidad en el discurso.

"Si el proceso", por ejemplo, "se representa como autoengendrado no hay agente; si se lo representa como engendrado por una causa externa aparece el participante que funciona como agente" (Ghio \& Fernández, 2008: 118).

Una cláusula como 'Los anuncios se desactivaron' está estructurada como medio + proceso,

"de manera que en el mundo real pudo haber un agente externo involucrado [en la desactivación de los anuncios], pero en la semántica 
ha sido representado este evento como autogenerado" (Ghio \& Fernández, 2008: 118).

Podemos, así, comparar 'Los anuncios se desactivaron' vs. 'Los anuncios fueron desactivados' y observar cómo la agentividad se manifiesta de manera diferente en ambas estructuras, aunque en ninguna de las dos aparezca el agente explícito:

“el último ejemplo tiene implícito el rasgo de agencia de manera que uno puede preguntar por quién, mientras que el primero solo permite un participante" (Ghio \& Fernández, 2008: 120).

Lavid et al. (2010) muestran que la función experiencial actor/agente puede ser atribuida al 'se' pasivo y al impersonal en español. Esta codificación, nuevamente, es muy relevante cuando analizamos grados de responsabilidad, pues en ella la agencia queda absolutamente desdibujada: no solo indefinida -como sucedería en un caso como 'Desactivaron los anuncios'- sino prácticamente borrada.

\subsection{Responsabilidad - Modo y modalidad}

La función interpersonal, por su parte, captura la dimensión pragmática del lenguaje como interacción, entendida como el intercambio de informaciones o de bienes y servicios entre al menos dos hablantes en el que el iniciador toma el rol de dar o solicitar y ubica a su/s interlocutor/es en el rol de reaccionar de alguna manera (según lo esperado o no). Los sistemas de modo (Mood) y modalidad (modality) brindan las opciones para realizar la función discursiva de la cláusula, definir su polaridad afirmativa o negativa y también para atemperar los mensajes con el fin de atenuarlos o matizarlos (Halliday, 1994). El sistema de modalidad se subdivide en modalización, que regula el intercambio de informaciones, y modulación, que interviene en el intercambio de bienes y servicios (estos dos alcances de la modalidad se vinculan fuertemente a lo que la gramática tradicional denomina modalidad epistémica y deóntica respectivamente). En español, los recursos más relevantes involucrados en la realización de las distintas funciones discursivas incluyen la flexión verbal, la entonación, el sujeto gramatical y, en menor medida, el orden de palabras. La identificación del sujeto gramatical -a partir del número y persona de la flexión verbal (cuando el constituyente está elidido), del pronombre nominativo o del grupo nominal que lo realiza (cuando está explícito)- resulta particularmente relevante para nuestros intereses en tanto nos permite ver si "el elemento sobre el que recae la "responsabilidad modal", en donde se construye la validez del argumento" (Halliday, 2017 [1992]: 93) encuentra su referencia en quien se constituye como emisor de los mensajes que analizamos (la primera persona), en el que los recibe (la segunda persona, el destinatario) o en una tercera persona que queda fuera de la relación de interlocución. Entre las opciones vinculadas con la modalidad (modalización y modulación) se incluyen algunos tiempos y modos verbales, los verbos y adjuntos modales y ciertas opciones léxicas vinculadas al verbo (predicator). Se suman, además, 
las denominadas metáforas gramaticales de modalidad (Halliday \& Matthiessen, 2004), en las que el intercambio se modaliza o modula con una cláusula:

"They are classiffied as metaphorical because a modality that would usually be realized either as a Finite modal operator or an Adjunct in fact gets realized as a clause" (Eggins, 2004: 174).

La aparición de metáforas gramaticales en la modalización y/o la modulación es muy relevante, en tanto muchas de las causas que explican estas opciones atípicas, marcadas, se relacionan con la realización de significados orientados a 'manipular' la responsabilidad de los participantes involucrados. En el caso del intercambio de informaciones (dar información - declarar), la modalización regula los grados de certeza, probabilidad y/o frecuencia de la información intercambiada. Las opciones involucradas en la realización de modalización (modalidad epistémica) involucran verbos modales, algunos adjuntos modales, ciertos tiempos y modos verbales y metáforas gramaticales que marcan la presencia del hablante o, por el contrario, lo ocultan tras una formulación pretendidamente objetiva (del tipo 'Es posible que'). La modalización aparece claramente involucrada en la regulación del grado de responsabilidad del hablante que, siempre que modaliza, ya no afirma categóricamente sino que matiza la información intercambiada. En el caso de intercambios de bienes y servicios (dar cosas y acciones no verbales - ordenar/solicitar), las metáforas gramaticales de modulación (modalidad deóntica) permiten 'oscurecer', y hasta ocultar, la agentividad (Eggins, 2004). En una cláusula como 'Se solicita que los usuarios confirmen su clave' la agencia está oscurecida; la despersonalización deja a los interlocutores sin nadie a quien discutir la solicitud, la orden o la instrucción. Antes de poder reaccionar negativamente a la solicitud, el interlocutor deberá ser capaz de identificar o establecer quién es el que ordena o pide. Eggins (2004: 186) define estas metáforas gramaticales de modalidad como expresiones impersonales de actitudes personales -aunque la formulación revele una aparente contradicción: "it is a covert attempt to get people to do things without having to take responsibility for issuing the command".

\section{Marco metodológico}

Tal como señala la introducción, el objetivo de este trabajo es identificar estrategias discursivas vinculadas a la distribución de grados de responsabilidad entre los participantes de los mensajes automáticos a partir de una mirada que observe y registre la complementariedad entre las funciones de los elementos en la transitividad y el modo (que discutimos en la sección anterior). Una vez definido el corpus, recolectado y fijado en dos meses por la autora, la metodología empleada fue empírica y cualitativa: segmentamos cada uno de los textos del corpus en cláusulas y analizamos específicamente los verbos, entendidos como opciones efectivamente realizadas en recursos (Menéndez, 2006), y su alcance en tanto categoría que realiza los significados experiencial, como proceso, e interpersonal, como proceso conjugado (o no), 
mediante tablas analíticas diseñadas específicamente para esta investigación (que describiremos en la subsección 2.2). A partir de dichas tablas analíticas, establecimos dos sistemas de opciones disponibles: uno relacionado con el grado de agentividad de los roles definidos por los tipos de procesos y otro vinculado con la asignación de la referencia sobre esos roles. La combinación de dichos grados, como explicaremos en las siguientes secciones, muestra cómo se distribuye la responsabilidad sobre las acciones predicadas en los textos entre el emisor institucional -sujeto discursivo que reparte los roles vinculados con la agentividad-y el destinatario del mensaje.

\subsection{Corpus}

Las interacciones que forman parte del corpus comparten tres características. En primer lugar, su motivación es práctica. Martin (2000) define, frente a interacciones que proyectan la expansión (como las conversaciones casuales), interacciones que proyectan el cierre, orientadas a un objetivo concreto que, una vez alcanzado, clausura el intercambio. Las interacciones del corpus corresponden a este último tipo. En términos de Martin (2000), se trata de interacciones pragmáticas. En segundo lugar, comparten la naturaleza de los participantes: el emisor es institucional, el destinatario no. Este tipo de mensajes se caracterizan por sostener una pretendida interacción 'uno a uno', aunque mientras que el destinatario del mensaje automático es efectivamente un individuo con una identidad definida, el emisor es siempre institucional representante de una empresa de servicios, comercial, de instituciones educativas, etc. En tercer lugar, se trata de mensajes automáticos, es decir, preprogramados; funcionan con una lógica propia y cerrada que desconoce, por ejemplo, ciertas variables del contexto situacional que podrían modificar la respuesta. El participante 'humano' se encuentra entonces con un mensaje automático, preprogramado, que lo sujeta a un número finito y limitado de acciones posibles.

Conformamos un corpus de mensajes automáticos compuesto por veintinueve correos electrónicos que tienen en común el remitente 'no-responder' (primera limitación para el participante individual que se encuentra restringido a no contestar, lo que constituye una restricción discusiva pero también material) -Subcorpus A-, diecisiete mensajes de texto telefónicos provenientes de números a los que tampoco pueden enviarse respuestas (el sistema las rechaza con un error) -Subcorpus B- y veinte transcripciones de contestadores automáticos (en los que para continuar con el motivo de nuestra llamada -por lo general, un reclamo o consulta- estamos obligados a seguir la lógica de las opciones habilitadas en el contestador) -Subcorpus C. Las interacciones orales del subcorpus $\mathrm{C}$ fueron grabadas mediante una aplicación que permite la captura de la voz y transcriptas según las siguientes pautas: |: pausa breve; $<x$ segs $>$ : pausas de más de 3 segs; /: entonación ascendente; \: entonación descendente; :: alargamiento de sonido vocálico; -: interrupción, MAYÚSCULAS: elevación de la voz, ?: entonación interrogativa; !: entonación enfática; (versales): 
comentarios de la transcriptora; (...): fragmentos omitidos. En todos los casos, modificamos los nombres de los participantes mencionados para mantener el anonimato y, en algunos, también de las empresas e instituciones.

\subsection{Diseño de investigación y materiales}

El primer paso de la investigación consistió en analizar cada uno de los textos del corpus. Para desarrollar dicho análisis, diseñamos tablas conformadas por cinco columnas: la primera columna incluye la cláusula (numerada), en la que destacamos en negrita el proceso que la define (para la segmentación en cláusulas tomamos como criterio la presencia de un proceso); en la segunda columna, clasificamos el proceso según su tipo, grado de concreción y según el participante activo que dicho proceso habilita (sujeto lógico para Halliday (1975)) en función de su tipo y su grado de concreción; en la columna tres, contrastamos las realizaciones específicas de la agentividad en cada cláusula en tanto parte de un discurso: señalamos cuál es, en caso de que hubiere, el participante activo y explicitamos su referencia; finalmente, en las columnas cuatro y cinco relevamos realizaciones vinculadas con el modo y la modalidad: sujetos gramaticales, funciones discursivas, modalización o modulación (si corresponde). A continuación, presentamos como ejemplo el caso 2 del subcorpus A (de correos electrónicos), seguido de la Tabla 2 mediante la que desarrollamos su análisis.

Subcorpus A- Caso 2)

El saldo de tu cuenta publicitaria está vacío

Hola María José:

El saldo de la cuenta publicitaria María José García está vacío. Por este motivo, se desactivaron todos los anuncios activos. Agrega dinero para volver a activarlos o para crear nuevos anuncios.

\section{AGREGAR DINERO VER CUENTA}

Gracias,

El equipo de publicidad de Facebook

No respondas a este mensaje porque es automático. Si tienes preguntas sobre los anuncios, puedes obtener ayuda. También puedes administrar la configuración de notificaciones por correo electrónico para esta cuenta publicitaria. 
Tabla 2. Análisis caso 2, subcorpus A.

\begin{tabular}{|c|c|c|c|c|}
\hline Cláusula & $\begin{array}{c}\text { Grado de concreción } \\
- \\
\text { Tipo de proceso } \\
\text { SUJETO LÓGICO }\end{array}$ & $\begin{array}{c}\text { Agentividad: } \\
\text { (Participante activo - } \\
\text { referencia) }\end{array}$ & $\begin{array}{l}\text { Modo }(M o o d) \\
\text { Sujeto } \\
\text { gramatical }\end{array}$ & $\begin{array}{c}\text { Modalidad } \\
\text { modalización } \\
\text { / } \\
\text { modulación }\end{array}$ \\
\hline $\begin{array}{l}\text { 1- El saldo de } \\
\text { tu cuenta } \\
\text { publicitaria } \\
\text { está vacío }\end{array}$ & 0 - relacional & -- & -- & Declarativa \\
\hline $\begin{array}{l}\text { 2- Hola María } \\
\text { José: cláusula } \\
\text { menor }\end{array}$ & -- & $\begin{array}{ll}- \\
-\end{array}$ & -- & -- \\
\hline $\begin{array}{l}\text { 3- El saldo de } \\
\text { la cuenta } \\
\text { publicitaria } \\
\text { María José } \\
\text { García está } \\
\text { vacío }\end{array}$ & 0 - relacional & -- & & Declarativa \\
\hline $\begin{array}{l}\text { 4- por ese } \\
\text { motivo, se } \\
\text { desactivaron } \\
\text { todos los } \\
\text { anuncios } \\
\text { activos }\end{array}$ & $\begin{array}{l}\text { IV - material } \\
\text { (desactivar) } \\
\text { ACTOR }\end{array}$ & $\begin{array}{l}\text { MEDIO + proceso } \\
{[-\mathrm{Ag}]}\end{array}$ & $\begin{array}{l}\text { Todos los } \\
\text { anuncios } \\
\text { activos } \\
\text { (Suj=Meta/Af) }\end{array}$ & Declarativa \\
\hline $\begin{array}{l}\text { 5- agrega } \\
\text { dinero para } \\
\text { volver a } \\
\text { activarlos }\end{array}$ & $\begin{array}{l}\text { IV - material (agregar) } \\
\text { ACTOR }\end{array}$ & ACTOR (destinatario) & $\begin{array}{l}<\text { tú> } \\
\text { (Ref: "MJG", } \\
\text { cláusula menor } \\
\text { 2) }\end{array}$ & Apelativa \\
\hline $\begin{array}{l}\text { 6- agregar } \\
\text { dinero }\end{array}$ & $\begin{array}{l}\text { IV - material (agregar) } \\
\text { ACTOR }\end{array}$ & ACTOR (destinatario) & -- & $\begin{array}{l}\text { Apelativa } \\
\text { (no personal) }\end{array}$ \\
\hline 7- ver cuenta & $\begin{array}{l}\text { I - mental (ver) } \\
\text { PROCESADOR }\end{array}$ & $\begin{array}{l}\text { PROCESADOR } \\
\text { (destinatario) }\end{array}$ & -- & $\begin{array}{l}\text { Apelativa } \\
\text { (no personal) }\end{array}$ \\
\hline $\begin{array}{l}8-\text { Gracias } \\
([<\text { te damos } \\
\text { las }>\text { gracias }])\end{array}$ & $\begin{array}{l}\text { II - verbal (agradecer) } \\
\text { DICENTE }\end{array}$ & DICENTE (emisor) & -- & Declarativa \\
\hline $\begin{array}{l}\text { 9- no } \\
\text { respondas } \\
\text { este mensaje } \\
\text { porque es } \\
\text { automático }\end{array}$ & $\begin{array}{l}\text { II - verbal } \\
\text { (responder) } \\
\text { DICENTE }\end{array}$ & $\begin{array}{l}\text { DICENTE } \\
\text { (destinatario) }\end{array}$ & $<$ tú $>$ & Apelativa \\
\hline $\begin{array}{l}10-(\text { Si tienes } \\
\text { preguntas } \\
\text { sobre los } \\
\text { anuncios) } \\
\text { puedes } \\
\text { obtener ayuda } \\
\text { ([obtener= } \\
\text { buscar] })\end{array}$ & $\begin{array}{l}\text { IV - material } \\
\text { (obtener=buscar) } \\
\text { ACTOR }\end{array}$ & ACTOR (destinatario) & $<$ tú $>$ & $\begin{array}{l}\text { Apelativa } \\
\text { Modulación } \\
\text { (Puedes } \\
\text { obtener) }\end{array}$ \\
\hline $\begin{array}{l}\text { 11- puedes } \\
\text { administrar la } \\
\text { configuración } \\
\text { de mensajes }\end{array}$ & $\begin{array}{l}\text { IV - material } \\
\text { (administrar) } \\
\text { ACTOR }\end{array}$ & ACTOR (destinatario) & $<$ tú $>$ & $\begin{array}{l}\text { Apelativa } \\
\text { Modulación } \\
\text { (Puedes } \\
\text { administrar) }\end{array}$ \\
\hline
\end{tabular}




\subsection{Procedimiento}

La tabla analítica propuesta despliega la complementariedad de las realizaciones experienciales (transitividad) e interpersonales (modo): la observación horizontal de los recursos analizados en cada columna de la tabla permite establecer cruces interesantes vinculados con la definición y la interpretación de la manera en que se distribuyen los grados de responsabilidad entre los participantes de la interacción. Así, la observación contrastiva de las columnas 2 y 3 , por ejemplo, releva la explicitación o el ocultamiento del participante que hace, dice o experimenta: la columna 3 muestra la realización semántica concreta del evento en el discurso, más allá de los roles inherentes habilitados por el tipo de proceso, señalados en la columna 2. Dicho contraste pone 'blanco sobre negro' la opción tomada por el hablante/escritor en tanto permite ver si el evento material, por ejemplo, se presenta como resultado de la acción de un participante o como autoengendrado, y si dicha acción es voluntaria (lo que configura un participante ACTOR) o instigada por otra entidad (que configura apenas un EJECUTOR). Las columnas 3 y 4, por su parte, muestran si el rol activo encuentra su referencia en alguno de los participantes involucrados en la interacción: en la primera persona, sujeto discursivo emisor del mensaje, o en la segunda persona que lo recibe, el destinatario. En diálogo con dicho análisis definimos, entonces, conjuntos de opciones disponibles desplegadas en dos continum de grado que van de la mayor atribución de responsabilidad (Grado 5) a la menor (Grado 1) con relación a la agentividad (Opciones disponibles para la realización de la agentividad) y con relación al modo (Opciones disponibles para la identificación referencial del participante activo, el emisor y/o el destinatario). La realización conjunta de esas opciones, entendemos, construye las estrategias orientadas a regular, aumentar o disminuir, la responsabilidad de quienes participan de estas interacciones.

Atribución de grados de responsabilidad (de mayor -grado 5- a menor -grado 1) en la transitividad - Opciones disponibles para la realización de la agentividad:

Ag- Grado 5- Explicitación del participante activo, que hace y dice. Definición del participante que hace y dice como ACTOR, CONDUCTOR (causantes de la actividad material) o DICENTE (fuente de la actividad verbal).

Ag- Grado 4- Explicitación del participante activo, que hace y dice. Definición del participante que hace y dice como EJECUTOR y TRANSMISOR respectivamente (participantes que hacen y dicen peropor causa de otro).

Ag- Grado 3- Explicitación del participante activo que experimenta, reacciona, percibe el evento, pero no hace - i.e PROCESADOR.

Ag- Grado 2- Ocultamiento del participante activo o agente (el evento se construye como autoengendrado):MEDIO+ proceso [-Agente]. 
Ag- Grado 1- Construcción del evento sin agentividad (participantes caracterizados, identificados o cuya existencia se marca mediante procesos relacionales y existenciales [-activos]).

Observamos a continuación la identificación de la referencia de los roles analizados en la transitividad con alguno de los interlocutores concretos que participan de la interacción: el emisor -sujeto discursivo construido como productor del mensaje- y/o el destinatario del mensaje automático:

Opciones disponibles para la identificación referencial del participante activo atribución de grados de responsabilidad (de mayor -grado 5- a menor -grado 1) según el modo:

Mo- Grado 5- El participante activo es identificado con alguno de los interlocutores, emisor $\mathrm{y} / \mathrm{o}$ destinatario, como participante individual, no institucional: sujeto gramatical en $1 \mathrm{ra}$ o $2 \mathrm{da}$ persona singular (designan respectivamente a emisor y/o destinatario, con una identidad individualizada).

Mo- Grado 4- El participante activo es identificado con alguno de los interlocutores, emisor y/o destinatario, como participante colectivo, institucional: sujeto gramatical en 1 ra persona plural no inclusiva o 2 da persona plural (designan respectivamente a emisor y/o destinatario, con una identidad institucional).

Mo- Grado 3- El participante activo involucra a alguno de los interlocutores pero de manera inespecífica y general: primera persona plural inclusiva, infinitivo.

Mo- Grado 2- El participante activo no es identificado con ninguno de los interlocutores (ni emisor ni destinatario): sujeto gramatical en 3ra persona singular o plural.

Mo- Grado 1: El participante activo no es identificado. 1.2vozpasivaser+participio (sin Complemento Agente); 1.1 "se pasivo" o "se impersonal".

Una vez definidas ambas escalas, analizaremos el ejemplo introducido anteriormente (caso 2 del Subcorpus A). El análisis cualitativo desarrollado a partir de la Tabla 2 permite relevar cómo se distribuye la responsabilidad entre los interlocutores: el emisor -remitente, que envía el e-mail- y el destinatario -que recibe el correo en su cuenta. Indicamos entre paréntesis qué opciones de agentividad (Ag) y modo (Mo) son combinadas en cada caso cuando las actividades involucran al destinatario o al emisor. En este caso, el correo electrónico es enviado como consecuencia de un inconveniente: el saldo de la cuenta de la usuaria de Facebook, destinataria del mail, estaba vacío por lo que sus anuncios activos se encontraban, como consecuencia, desactivados. Ahora bien, la aparición del problema que justifica dicho correo electrónico no tiene responsables claramente identificados: las dos primeras cláusulas introducen un estado de cosas cuya responsabilidad no se atribuye a la acción de ningún participante (Ag-Grado 1): las cláusulas 1 y 3 son relacionales, 
predican un estado ('vacío') de 'el saldo de la cuenta publicitaria' del destinatario y funcionan discursivamente como justificación del mail y de las acciones, materiales y verbales, puestas en juego. La cláusula 4, por su parte, introduce la consecuencia derivada: "por este motivo se desactivaron todos los anuncios activos". El actoragente aparece desdibujado, la cláusula se estructura como medio + proceso $[-\mathrm{Ag}]$ (Ag- Grado 2+ Mo- Grado 1). Como señalábamos más arriba, en el evento 'real' pudo haber un agente externo involucrado en la desactivación de los anuncios pero en la cláusula dicho agente es borrado (a partir de la pasiva con se) y el evento se construye como autoengendrado. Ahora bien, la solución del inconveniente se presenta como responsabilidad exclusiva de quien recibe el correo, i.e. del destinatario: seis son las cláusulas agentivas en las que el sujeto lógico -agente de la actividad material o verbal en cuestión- refiere al destinatario: explícitamente designado por la segunda persona singular en agrega (5), (no)respondas (9), puedes obtener (10), puedes administrar (11) (Ag- Grado 5 + Mo- Grado 5) o implicado en los infinitivos agregar y ver (6 y 7 ) (AgGrado 3 y 5 + Mo- Grado 3). Sujeto lógico y sujeto gramatical (Halliday, 1975) coinciden y se explicitan claramente cuando la responsabilidad de la actividad se atribuye al destinatario del mail (cláusulas 5, 9, 10 y 11). Mientras que el problema que motiva la interacción carece de responsables, la solución del inconveniente se presenta como responsabilidad exclusiva del participante individual que recibe el correo.

Esta tendencia domina los casos estudiados: el análisis de las opciones de agentividad y modo realizadas muestra que se construyen altos grados de responsabilidad sobre las acciones predicadas atribuida a los destinatarios de los mensajes pero grados bajos atribuidos a los emisores. El subcorpus B, como señalamos en la sección 2.1 , está conformado por mensajes de texto recibidos en el teléfono móvil. Como ejemplo, presentamos el caso 1, un mensaje de la empresa Movistar (remitente que suele estar explicitado), 'disparado' toda vez que se realiza el pago de la factura mensual de la línea involucrada. ${ }^{5}$

$\mathrm{B}-1)$

Recibimos un pago por $\$ 644.05$. Si abonaste el total de tu factura y tu línea se encuentra interrumpida, se rehabilitará en las próximas $2 \mathrm{hs}$.

En el caso B-1), son dos las cláusulas principales, y las dos son agentivas. Es interesante observar el modo en que se relacionan los cuatro procesos de este texto: la

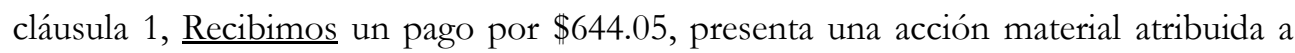
una primera personal plural (Ag- Grado 5 + Mo- Grado 4) que acredita la acción anterior (el pago) de otro participante, no especificado. Ahora bien, esa cláusula en modalidad declarativa no termina de desligar al destinatario del mensaje (aún cuando ya pagó) de la responsabilidad sobre futuras acciones ya que la cláusula siguiente introduce una situación hipotética en pasado en la que, una vez más, la agentividad es claramente atribuida al destinatario pero aparece desdibujada cuando el evento se relaciona con el emisor: $2.1-\mathrm{Si}<\mathrm{vos}>$ abonaste el total de tu factura [y] (Ag- Grado $5+$ 
Mo- Grado 5) 2.2- tu línea se encuentra interrumpida (Ag- Grado 2 + Mo- Grado 1), 2- se rehabilitará en las próximas dos horas (Ag- Grado $2+$ Mo- Grado 1). La interrupción de la línea no es atribuida a ningún agente -la cláusula predica un estado de cosas del sujeto gramatical pasivo 'tu línea' (se encuentra interrumpida), sin mencionar la causa o el motivo que provoca dicha interrupción- y tampoco se identifica al responsable de su rehabilitación -al estructurar las cláusulas como medio+proceso, con las pasivas con se. ¿Por qué las cláusulas son codificadas de esta manera y no de otra? La obra de Halliday ha mostrado claramente que "describing and relating are one operation, not two" (Halliday, 2008: 123). En este sentido, y para responder la pregunta anterior, resulta operativo confrontar los recursos efectivamente realizados con otras opciones, posibles en español aunque no tomadas, que darían lugar a estructuras del tipo 'si tu factura fue abonada e interrumpimos tu línea, la rehabilitaremos en dos horas', o 'tu línea ha sido interrumpida pero Movistar la rehabilitará en dos horas' entre muchísimas otras posibilidades. Sobre la base de lo que pudo haber sido y no fue podemos explicar las opciones tomadas en este caso concreto, orientadas, claramente, a desdibujar la agencia del remitente institucional. Como muestra la combinación de opciones vinculadas a la agentividad y el modo ni el responsable del problema, la posible interrupción de la línea, ni el responsable de su rehabilitación son identificados.

El último de los ejemplos forma parte del subcorpus $\mathrm{C}$ de contestadores automáticos; es el mensaje que recibe a quienes se comunican con el centro de atención al cliente de una empresa proveedora de energía eléctrica.

C-1)

Usted se ha comunicado con el centro de atención telefónica de EDEA \EDEA informa que debido a un desperfecto en una línea de media tensión se encuentra afectado en forma parcial | el suministro de energía eléctrica en los siguientes barrios || José Hernández \Ostende \ Newbery \ibertad $\backslash$ y Ameghino \|| se estima su normalización aproximadamente a la hora trece y treinta \por cualquier otra consulta aguarde| y será atendido $\backslash \|||$ por consultas trámites y reclamos comerciales marque $1 \backslash \mid$ por emergencias e inconvenientes técnicos en el servicio eléctrico marque 2 | por denuncias de conexiones ilegales marque $3 \backslash$

Las llamadas que están en el origen del subcorpus $C$ se justifican por la necesidad de hacer una consulta o un reclamo. Sin embargo, el reclamo o la consulta aparecen generalmente mediados por un contestador automático. Ocho son las cláusulas principales en el caso C-1), todas agentivas. El emisor se identifica en la cláusula 2 como dicente, fuente de la información que suscita la llamada del usuario-'EDEA informa que'- pero se construye como un participante colectivo, la empresa (AgGrado 5 +Mo- Grado 4). EDEA se hace responsable de la información del desperfecto técnico que ocasiona el inconveniente pero desdibuja su responsabilidad en la causa del problema material y en su solución: 2.1- 'debido a un desperfecto en 
una línea de media tensión se encuentra afectado en forma parcial el suministro de energía eléctrica en los siguientes barrios', 3- 'se estima su normalización aproximadamente a las trece y treinta'. Ambas cláusulas desdibujan la participación de un agente: la primera atribuyendo a un 'desperfecto' el deterioro del suministro de electricidad, la segunda evitando identificar el participante que se hace cargo de la estimación del tiempo restante para su rehabilitación, cláusula estructurada como medio+ proceso, a partir de la pasiva con se (Ag- Grado $2+$ Mo- Grado 1). La tercera de las cláusulas en la que la agentividad cae del lado del emisores la 5, 'será atendido', cláusula pasiva con ser en la que no se especifica el agente que se hará cargo en caso de necesitar resolver otra consulta (Ag- Grado $5+$ Mo- Grado 1). El interlocutor de la llamada se esconde: o porque el evento se presenta como un estado de cosas, o como autoengendrado (mediante la estructura de la pasiva con se), o porque no se especifica su identidad (a partir de la pasiva sin complemento agente), o porque se trata de un participante colectivo (EDEA). La persona capaz de asistirnos en el reclamo no es identificada y, por lo tanto, tampoco se concretiza. Frente a dicho panorama, cinco son las cláusulas que involucran al usuario que llama como participante activo que hace o dice, referencia que se explicita en la realización del sujeto gramatical, segunda persona singular formal <usted > (Ag- Grado $5+$ Mo- Grado 5): la primera cláusula, en modalidad declarativa -usted se ha comunicado con Edea (dicente, cláusula 1), confirma la llamada; las cuatro restantes, en modalidad apelativa, demandan una acción material del usuario -'aguarde' (cláusula 4, actor) y 'marque' (cláusulas 6, 7 y 8, actor). La consulta o reclamo se prolongan, y las decisiones sobre qué hacer en pos de la solución de la consulta deben ser tomadas por el usuario del servicio.

\section{Resultados}

La tendencia que muestra el análisis de los tres casos presentados en la sección anterior es representativa. La Tabla 3 muestra qué combinaciones predominan en la realización de la agentividad (Ag), por un lado, y de la identificación referencial del participante activo (Mo), por otro, cuando las actividades representadas en las cláusulas involucran la participación del destinatario (i.e. de quienes reciben los correos y mensajes de texto, y de quienes se encuentran con el contestador automático) y cuando no lo involucran: 
Tabla 3. Combinación de opciones que predomina en cada subcorpus.

\begin{tabular}{|l|l|l|}
\hline & \multicolumn{1}{|c|}{$\begin{array}{c}\text { Actividad } \\
\text { +Destinatario }\end{array}$} & \multicolumn{1}{c|}{$\begin{array}{c}\text { Actividad } \\
\text {-Destinatario }\end{array}$} \\
\hline Subcorpus A & $\begin{array}{l}\text { I) Ag- Grado 5 + Mo- Grado 5 } \\
\text { II) Ag- Grado 5 + Mo- Grado 3 }\end{array}$ & $\begin{array}{l}\text { I) Ag- Grado 2 + Mo- Grado 1 } \\
\text { II) Ag- Grado 1 } \\
\text { III) Ag- Grado 5 + Mo- Grado 4 }\end{array}$ \\
\hline Subcorpus B & I) Ag- Grado 5 + Mo- Grado 5 & $\begin{array}{l}\text { I) Ag- Grado 2 + Mo- Grado 1 } \\
\text { II) Ag- Grado 1 } \\
\text { III) Ag- Grado 5 + Mo- Grado 4 }\end{array}$ \\
\hline Subcorpus C & I) Ag- Grado 5 + Mo- Grado 5 & $\begin{array}{l}\text { I) Ag- Grado 2 + Mo- Grado 1 } \\
\text { II) Ag- Grado 5 + Mo- Grado 1 } \\
\text { III) Ag- Grado 5 + Mo- Grado 4 }\end{array}$ \\
\hline
\end{tabular}

Los resultados son elocuentes. Cuando las actividades representadas en los textos involucran al destinatario, la combinación que predomina -opción exclusiva en los subcorpus B y C- es la que identificamos como Ag- Grado $5+$ Mo- Grado 5: el participante activo que hace o dice se explicita y se lo construye como causa o fuente de la actividad material o verbal respectivamente y dicho participante -agente- se identifica, además, con el destinatario del texto en cuestión, a quien se personaliza e individualiza. ${ }^{6}$ Ahora bien, cuando las actividades representadas en las cláusulas no involucran al destinatario, el agente se desdibuja o se borra: los eventos se construyen, en la mayoría de los casos, como autoengendrados o como realidades en las que no intervienen participantes activos. Según muestra la Tabla 3, la combinación que predomina en los tres subcorpus es Ag-Grado $2+$ Mo- Grado 1, es decir, la opción por el ocultamiento del participante activo (a la que caracterizamos con el grado 2 de Agentividad) en cláusulas que se estructuran como medio+proceso, sin agente, realizadas mediante el se pasivo en español (opción que caracterizamos como el grado 1 del Modo). En segundo lugar, esta vez en los subcorpus A y B, aparece fuertemente la opción por el grado 1 de Agentividad, esto es, casos en los que el evento se construye sin roles activos, mediante procesos relacionales y existenciales en los que se caracterizan o identifican participantes, o cuya existencia se marca, pero sin que medie la acción de ningún otro participante. Finalmente, en el tercer lugar, aparecen casos de explicitación del participante activo que hace o dice (i.e. Ag-Grado 5) identificado con el emisor pero, en dichos casos, la construcción del responsable siempre es institucional, colectiva, realizada a partir de la primera persona plural no inclusiva (i.e. Mo- Grado 4). El análisis de dichos recursos muestra, entonces, la construcción de altos grados de responsabilidad sobre las acciones predicadas atribuida al destinatario de los mensajes pero grados bajos atribuidos a quien los emite. La distribución de la responsabilidad resulta, entonces, muy desigual: el peso cae sobre el lado del destinatario/oyente de los mensajes quien debe decidir cuál es el problema que tiene y qué hacer en consecuencia (aún cuando el expertise sea del otro). Este tipo de textos, entonces, no dejan demasiado espacio para la responsabilidad sea negociada. 


\section{Discusión}

El análisis desarrollado comprueba la hipótesis propuesta en la introducción: en el caso de los mensajes automáticos, los altos grados de agentividad asignada al destinatario de los mensajes y los grados muy bajos de agentividad del lado del emisor institucional explican el efecto discursivo, sugerido por numerosos testimonios recopilados por la autora, de estar participando de interacciones en las que la responsabilidad de decidir y de hacer recae exclusivamente sobre el participante no institucional. La estrategia discursiva orientada a personalizar al participante activo cuando este coincide con el destinatario e impersonalizarlo cuando refiere al emisor domina claramente los casos del corpus y, creemos, permite comenzar a caracterizar este tipo de mensajes. No obstante, el corpus con el que trabajamos es acotado. Sería deseable extender el alcance de esta investigación con el análisis de muestras más amplias para verificar si se mantienen las realizaciones dominantes identificadas en este trabajo.

Por otro lado, creemos que las dos escalas que proponemos (a las que identificamos como Agentividad y Modo por involucrar opciones de los sistemas de transitividad y modo respectivamente) pueden ser empleadas, con modificaciones, para analizar cómo se distribuye y se negocia la responsabilidad entre los interlocutores en otros corpus, en otros textos, en otros géneros discursivos.

\section{CONCLUSIONES}

Lavandera (2014 [1981]: 114) analiza la tensión entre lo impersonal y lo personal en la organización del discurso y señala que "un recurso común en la organización del discurso lo constituyen los cambios entre lo impersonal, general e indefinido, y lo personal, particular y definido". En el caso de los textos que conforman nuestro corpus, la estrategia dominante consiste en personalizar -particularizar y definir- al destinatario e impersonalizar -generalizar y no definir- al emisor, con el objetivo de responsabilizar al primero y quitarle responsabilidad al segundo. Los resultados presentados muestran una tendencia contundente en relación con los recursos realizados: la combinación preferida incluye Ag- grado 5 y Mo- grado 5 cuando la actividad involucra al destinatario, a quien se personaliza e individualiza. Por el contrario, cuando la actividad involucra al emisor, las combinaciones muestran grados bajos de agentividad y de referencia: el agente se desdibuja o desaparece. En los discursos analizados, entonces, el elemento que mueve estratégicamente la aguja de la personalización a la impersonalización en la organización discursiva es el cambio de participante de la interacción; la estrategia se orienta, así, a cargar de responsabilidad a uno -el destinatario- y limpiar de responsabilidad al otro participante -el emisor-, situación que explica la sensación de estar hablando solo a pesar de estar formando parte de una 'inter' acción. 


\section{REFERENCIAS BIBLIOGRÁFICAS}

Eggins, S. (2004). An Introduction to Systemic Functional Grammar. 2nd Edition. Nueva York/Londres: Continuum.

Ghio, E. \& Fernández, M. D. (2008). Lingüística sistémicofuncional. Aplicaciones a la lengua española. Santa Fe: UNL.

Halliday, M. A. K. (1975). Estructura y función del lenguaje. En J. Lyons (Ed.), Nuevos horizontes de la lingüistica (pp. 145-176). Madrid: Alianza Editores.

Halliday, M. A. K. (2017 [1992]). Algunos rasgos léxico gramaticales del texto de Crecimiento Poblacional Cero. En E. Ghio, F. Navarro \& A. Lukin (Eds.), Obras esenciales de M.A.K. Halliday (pp. 79-112). Santa Fe: Eudeba, UNL.

Halliday, M. A. K. (1994). An Introduction to Functional Grammar, 2nd edition. Londres: Edward Arnold.

Halliday, M. A. K. (2008). Complementarities in language. Beijing: The Commercial Press.

Halliday, M. A. K. \& Matthiessen, Ch. (2009). Systemic Functional Grammar: A first step into the theory. Beijing: Higher Education Press.

Halliday, M. A. K. \& Matthiessen, Ch. (2004). An Introduction to Functional Grammar, 3rd Edition. Londres: Edward Arnold.

Halliday, M. A. K. \& Webster, J. (Eds.) (2009). Continuum companion to systemic functional linguistics. Londres/Nueva York: Continuum.

Lavandera, B. (2014[1981]). Tensión entre lo personal y lo impersonal en la organización del discurso. En S. M. Menéndez (Ed.), Variación y significado. Y discurso (pp. 111-138). Buenos Aires: Paidós.

Lavandera, B. (2014 [1992]). Argumentatividad y discurso. En S. M. Menéndez (Ed.), Variación y significado. Y discurso (pp. 347-365). Buenos Aires: Paidós.

Lavid, J., Arús, J. \& Zamorano, J. R. (2010). Systemic Functional Grammar of Spanish. A contrastive study with English. Londres/Nueva York: Continuum.

Martin, J. (2000). Factoring out exchange: Types of structures. En M. Coulthard (Ed.), Working with Dialogue (pp. 19-40). Tubingen: Niemeyer.

Menéndez, S. M. (2000). Estrategias discursivas: Principio metodológico para el análisis pragmático del discurso. En J. J. de Bustos \& P. Chareadeau (Eds.), Lengua, discurso, texto. I Simposio Internacional de Análisis del discurso (pp. 926-945). Madrid: Visor. 
Menéndez, S. M. (2005). ¿Qué es una estrategia discursiva? En S. Santos \& J. Panesi (Comp.), Actas del Congreso Internacional: Debates Actuales. Las teorías críticas de la literatura y la lingüistica. Buenos Aires: Facultad de Filosofía y Letras (Edición en CD).

Menéndez, S. M. (2006). ¿Qué es una gramática textual? Buenos Aires: Littera.

Menéndez, S. M. (2013). La clasificación de los verbos en español: El principio de gradualidad. Un enfoque sistémico-funcional. En Actas del VII Congreso de la Asociación Internacional de Hispanistas. Buenos Aires: Facultad de Filosofía y Letras.

\section{NOTAS}

${ }^{1}$ A lo largo del trabajo usamos el término 'participante' con dos alcances que no se confunden entre sí: el primero, vinculado al sistema de transitividad, alude a los roles asignados por el proceso en cuestión; el segundo, relacionado con el sistema de modo, refiere a los interlocutores involucrados en las interacciones del corpus.

${ }^{2}$ Resulta significativa, no obstante, la ausencia de agentividad en los procesos relacionales y existenciales.

3 Solo cuestionaría este rasgo involuntario la sub clasificación de procesos mentales intencionales (Lavid et al., 2010) que interpretamos aquí como conductuales.

${ }^{4}$ Somos conscientes de que "not all processes expressing agency express also causation". En una cláusula como The baby's picking her nose again (Lavid et al., 2010: 86) interpretaríamos "the baby" como EJECUTOR, no ACTOR.

${ }^{5}$ Por razones de espacio, no incluimos las tablas analíticas de los dos ejemplos que siguen.

${ }^{6}$ Solo el 17\% de los casos presentan la opción de Mo-grado 3 en el subcorpus A -opción en la que el destinatario sigue siendo identificado con el participante activo pero de una manera inespecífica y general (a partir de la primera persona plural inclusiva o del infinitivo). 